\title{
Spin Hall and spin-diagonal conductivity in the presence of Rashba and Dresselhaus spin-orbit coupling
}

\author{
N. A. Sinitsyn, E. M. Hankiewicz, Winfried Teizer, and Jairo Sinova \\ Department of Physics, Texas A\&M University, College Station, Texas 77843-4242, USA \\ (Received 15 October 2003; revised manuscript received 28 May 2004; published 31 August 2004)
}

\begin{abstract}
We investigate the spin-current linear response conductivity tensor to an electric field in a paramagnetic two-dimensional electron gas with both Rashba and Dresselhaus spin-orbit coupling in the weak scattering regime within the Born approximation. In the usual sample parameters, and for cross-sectional size smaller than the spin-coherence length, the spin-Hall conductivity depends only on the sign of the difference in magnitude of the Rashba and Dresselhaus coupling except within a narrow window where both coupling strengths are equal. We also find that a spin current is generated in the direction of the driving field for a non-zero Dresselhaus term. Possible experimental setups for its detection, taking into account the finite mobility and typical parameters of current samples, are discussed.
\end{abstract}

DOI: 10.1103/PhysRevB.70.081312

The manipulation of spin by electrical means in semiconducting enviroments has generated a lot of recent theoretical and experimental research aimed at developing useful spintonic devices and novel physical concepts, ${ }^{1}$ many focusing on effects that generate spin-polarized current. ${ }^{2}$ Given the success of ferromagnetic metal based spintronic devices, ${ }^{3}$ which have revolutionized the information storage industry, the possibility of doping, gating, and heterojunction formation in seminconducting spintronic devices makes their possibilities that much wider. However, the practical implementation of semiconducting spintronics is awaiting the resolution of effective injection of spin-polarized carriers $^{4}$ from ferromagnetic metals combined with long spin lifetimes, ${ }^{5}$ or room-temperature semiconductor ferromagnetism. ${ }^{6}$ The recently proposed intrinsic spin-Hall effect by Murakami et al. ${ }^{7}$ in $p$-doped semiconductors and by Sinova et al. ${ }^{8}$ in Rashba spin-orbit coupled two-dimensional electron gases (2EDGs) offers new avenues in spintronics research and transport phenomena which may meet the first challenge.

The intrinsic spin-Hall effect consists of a dissipationless spin-current contribution generated perpendicular to the driving electric field whenever spin-orbit coupling is stronger than the scattering induced disorder and the spin-coherence length is larger than the cross-sectional system size. This effect contrasts with the proposed extrinsic spin-Hall effect recently revived by Hirsch ${ }^{9}$ and Zhang ${ }^{10}$ and first studied by Dyakonov and Perel, ${ }^{11}$ where spin-orbit dependent scattering from impurities can generate a Hall spin-current. In the Rashba spin-orbit coupled 2DEGs it was shown that the intrinsic spin-Hall conductivity has a value $e / 8 \pi$ in the case of both spin-split subbands being occupied. ${ }^{8}$ Motivated by recent experiments ${ }^{12,13}$ which have demonstrated the ability to tune the magnitude of the Rashba and Dresselhaus spin-orbit coupling strength directly and by the fact that when both coupling strengths are equal the quasiparticles are effectively spin-orbit decoupled ${ }^{14}$ and the intrinsic spin-Hall effect should vanish, we extend our prior studies to include 2DEGs with both Rashba and Dresselhaus spin-orbit coupling. We find that the spin Hall conductivity remains $e / 8 \pi$ in the weak scattering mesoscopic limit except for a small window where
PACS number(s): 73.43.-f, 72.25.Dc, 72.25.Hg, 85.75.- d

the spin-orbit coupling strengths are equal and changes sign when the difference of the spin-orbit coupling strengths changes sign. We also find a novel effect in which a spinpolarized current is generated in the direction of the driving electric field whenever the Dresselhaus spin-orbit coupling is nonzero, which should have important consequences if observed. ${ }^{15}$

The Hamiltonian which we consider contains the spinorbit coupling interaction form for a two-dimensional electron gas ${ }^{16}$

$$
H_{s o}=\frac{\lambda}{\hbar}\left(\hat{\sigma}_{x} \hat{p}_{y}-\hat{\sigma}_{y} \hat{p}_{x}\right)+\frac{\beta}{\hbar}\left(\hat{\sigma}_{x} \hat{p}_{x}-\hat{\sigma}_{y} \hat{p}_{y}\right),
$$

where the first term is the Bychkov-Rashba term due to the lack of inversion symmetry of the trapping well ${ }^{17}$ and the second is the linear Dresselhaus term due to the lack of inversion symmetry in bulk semiconductors. ${ }^{18}$ Hence the full particle Hamiltonian can be written as

$$
H=\left(\frac{\hat{p}^{2}}{2 m^{*}}-\mu\right) \sigma_{0}+\frac{\alpha_{i k}}{\hbar} \hat{\sigma}_{i} \hat{p}_{k}, \quad \alpha_{i k}=\left(\begin{array}{cc}
\beta & \lambda \\
-\lambda & -\beta
\end{array}\right) .
$$

This Hamiltonian has a simple spectra given by

$$
\xi_{ \pm}(\mathbf{k})=\frac{\hbar^{2} k^{2}}{2 m^{*}}-\mu \pm k \sqrt{\left(\lambda^{2}+\beta^{2}\right)+2 \lambda \beta \sin (2 \phi)},
$$

where $\tan \phi=k_{y} / k_{x}, \mu$ is the chemical potential, and the corresponding eigenfunctions are given by

$$
\psi_{ \pm}(\mathbf{r})=\frac{1}{\sqrt{2 A}}\left(\begin{array}{c}
e^{i \theta_{k} / 2} \\
\pm e^{-i \theta_{k} / 2}
\end{array}\right) e^{i \mathbf{k} \cdot \mathbf{r}},
$$

where

$$
\tan \theta_{k}=\frac{\lambda k_{x}+\beta k_{y}}{\lambda k_{y}+\beta k_{x}},
$$

and $A$ is the area of the system.

The Rashba coupling strength in a 2DEG can be modified by a gate field by up to $50 \%^{12,19}$ and therefore this system affords the study of the intricate interplay between both types of coupling directly whose ratio $\lambda / \beta$ can vary between 1.5 and 2.5 typically. ${ }^{13}$ Recent observations of a spin-galvaic 
effect $^{13,20}$ and spin-orbit coupling weak localization studies in these systems ${ }^{12,21,22}$ illustrate the potential importance of these tunable interactions in semiconductor spintronics. ${ }^{23}$ This intricate interplay has generated several theoretical studies of their transport properties ${ }^{16,24}$ and a new proposed spin-FET ${ }^{14}$ in the regime where $\lambda=\beta$ motivated by the original proposal by Datta and Das. ${ }^{25}$

In the Rashba coupled 2DEG $(\beta=0)$, Sinova et al. ${ }^{8}$ found that the $\mathrm{dc} z$-component spin-current Hall response to a driving internal electric field, $j_{s}^{z}=\sigma_{x y}^{\mathrm{spin}} E_{y}$, in the clean limit has a universal value whenever the two Rashba split bands are occupied (the usual case), $\sigma_{x y}^{\text {spin }}=e / 8 \pi$, and vanishes linearly with the electron density when only one Rashba split band is occupied. This result was obtained both within the Kubo linear response formalism and within an equivalent and more physically transparent multi-band wave-packet dynamics theory. $8,26,27$

To address the interplay between the Rashba and Dresselhaus spin-orbit coupling and its consequence on the spincurrent response to an applied electric field we use the linear response Kubo formalism. ${ }^{28}$ In this Kubo formalism approach the spin-current response to an electric field has the form

$$
\begin{aligned}
\sigma_{\alpha y}^{\text {spin }}= & e \hbar \sum_{n \neq n^{\prime}} \int \frac{d \vec{k}}{(2 \pi)^{2}}\left(f_{n^{\prime}, k}-f_{n, k}\right) \\
& \times \frac{\operatorname{Im}\left[\left\langle n^{\prime} k\left|\hat{j}_{\alpha}^{z}\right| n k\right\rangle\left\langle n k\left|\hat{v}_{y}\right| n^{\prime} k\right\rangle\right]}{\left(\xi_{n k}-\xi_{n^{\prime} k}\right)^{2}},
\end{aligned}
$$

where $n, n^{\prime}= \pm, \vec{j}^{z}=\hbar / 4\left\{\hat{\sigma}_{z}, \vec{v}\right\}, \vec{v}=\partial H(k) / \partial \hbar \vec{k}$, and $\alpha=x, y .^{23}$ We address the effects of finite quasiparticle lifetime, i.e., finite mobility samples, within the Born approximation (see below) to address the relevant experimental regime similarly to Refs. 29 and 30. This approximation captures the finite lifetime effects dues to disorder in a finite system size but still requires the spin-coherence length to be larger than the length of the spin transport considered since multiple scattering will eventually lead to loss of spin polarization in an infinite system size as shown in Ref. 31. Inserting eigenvalues, Eq. (3), and eigenvectors, Eq. (4), above we obtain (for the experimentally relevant regime of both subbands being occupied):

$$
\begin{aligned}
& \sigma_{x y}^{z}=\left\{\begin{array}{l}
\frac{e}{8 \pi} \text { for } \lambda^{2}>\beta^{2} \\
0 \text { for } \lambda=\beta \\
-\frac{e}{8 \pi} \text { for } \lambda^{2}<\beta^{2}
\end{array},\right. \\
& \sigma_{y y}^{z}=\left\{\begin{array}{l}
-\frac{e}{8 \pi} \frac{\beta}{\lambda} \text { for } \lambda^{2}>\beta^{2} \\
0 \text { for } \lambda=\beta \\
\frac{e}{8 \pi} \frac{\lambda}{\beta} \text { for } \lambda^{2}<\beta^{2}
\end{array} .\right.
\end{aligned}
$$

These equations predict that the spin-Hall conductivity only depends on the sign of $\beta^{2}-\lambda^{2}$, and that there is a diagonal (in the sense that it points along the driving field direction) reactive part of the spin-current whenever $\beta$ is non-zero and $\beta \neq \lambda$. The sharpness and singularities of these results are simply an artifact of the quasiparticle induced lifetime broadening $\eta \rightarrow 0$ limit, which can be easily rectified by introducing a finite lifetime to the spin-orbit coupled quasiparticles induced by the scattering in the usual Born approximation approach $^{32,33}$ given by

$$
\begin{aligned}
\sigma_{\alpha y}^{\mathrm{spin}}= & -\frac{e}{\omega} \int \frac{d \vec{k} d \epsilon}{(2 \pi)^{3}} \sum_{n n^{\prime}} f(\epsilon) \operatorname{Im}\left[\left\langle n^{\prime} \vec{k}\left|\hat{j}_{\alpha}^{z}\right| n \vec{k}\right\rangle\left\langle n \vec{k}\left|\hat{v}_{y}\right| n^{\prime} \vec{k}\right\rangle\right] \\
& \times\left[A_{n^{\prime}, \vec{k}}(\epsilon) \operatorname{Re}\left[G_{n, k}^{\mathrm{ret}}(\epsilon+\hbar \omega)\right]\right. \\
& \left.+A_{n, \vec{k}}(\epsilon) \operatorname{Re}\left[G_{n^{\prime}, k}^{\mathrm{adv}}(\epsilon-\hbar \omega)\right]\right],
\end{aligned}
$$

where $A_{n, \vec{k}}(\epsilon)=\eta /\left(\left(\epsilon-\xi_{n \vec{k}}\right)^{2}+\eta^{2} / 4\right)$ is the disorder broadened spectral function and $G_{n, k}^{\mathrm{ret} / \mathrm{adv}}(\hbar \omega)=1 /\left(\hbar \omega-\xi_{n, k} \pm i \eta / 2\right)$ are the advanced and retarded quasiparticle Green's functions with finite lifetime $2 \eta^{-1} / \hbar$ (here chosen to be momentum independent for simplicity). The above expression can be evaluated numerically, however, we can make progress understanding qualitatively the effect of finite quasiparticle lifetime by taking the further approximation that the spectral function is sharp enough to allow substituting it for a delta function. This simplification translates into adding a small complex value to the frequency in the frequency dependent response function from which the weak scattering dc limit expression, Eq. (6), was obtained. The expression that is obtained is identical to Eq. (6) but with the denominator replaced by $\eta^{2}+\left(\xi_{n k}-\xi_{n^{\prime}}\right)^{2}$. Anticipating already the typical experimental situation where the Fermi energy is only slightly modified from its non-spin-orbit coupled form, we obtain after some straightforward algebraic manipulation

$$
\sigma_{x y}^{\text {spin }}=\frac{e}{8 \pi} \frac{\epsilon_{F}\left(\epsilon_{\lambda}-\epsilon_{\beta}\right)}{\sqrt{\epsilon_{F}^{2}\left(\epsilon_{\lambda}-\epsilon_{\beta}\right)^{2}+\eta^{2} \epsilon_{F}\left(\epsilon_{\beta}+\epsilon_{\lambda}\right) / 4+\eta^{4} / 64}}
$$

and

$$
\begin{aligned}
\sigma_{y y}^{\mathrm{spin}}= & -\frac{e\left(\epsilon_{\beta}-\epsilon_{\lambda}\right)}{16 \pi \sqrt{\epsilon_{\beta} \epsilon_{\lambda}}} \\
& \times\left(1-\frac{\eta^{2}+8 \epsilon_{F}\left(\epsilon_{\lambda}+\epsilon_{\beta}\right)}{\sqrt{\eta^{4}+64 \epsilon_{F}^{2}\left(\epsilon_{\beta}-\epsilon_{\lambda}\right)^{2}+16 \eta^{2} \epsilon_{F}\left(\epsilon_{\beta}+\epsilon_{\lambda}\right)}}\right),
\end{aligned}
$$

where $\epsilon_{F}$ is the Fermi energy, and $\epsilon_{\lambda}=m \lambda^{2} / \hbar^{2}$ and $\epsilon_{\beta}$ $=m \beta^{2} / \hbar^{2}$ are the spin-orbit coupling characteristic energy scales for the Rashba and Dresselhauss mechanisms defined in the same way as Ref. 30, and $\epsilon_{F} \gg \epsilon_{\lambda}, \epsilon_{\beta}$ is assumed. In the limit of $\eta \rightarrow 0$ one recovers Eqs. (10) and (11), although near the $\alpha=\beta$ point we see that one limit goes smoothly but fairly rapidly to the next in the scale of $\eta$ as illustrated in Fig. 1. This analysis illustrates then that, unlike the universal Hall conductivity value on a 2DEG quantum Hall plateau, the universality of the intrinsic spin Hall effect does not extend to the disorder domain. Also, as expected, ${ }^{8}$ the spin Hall 


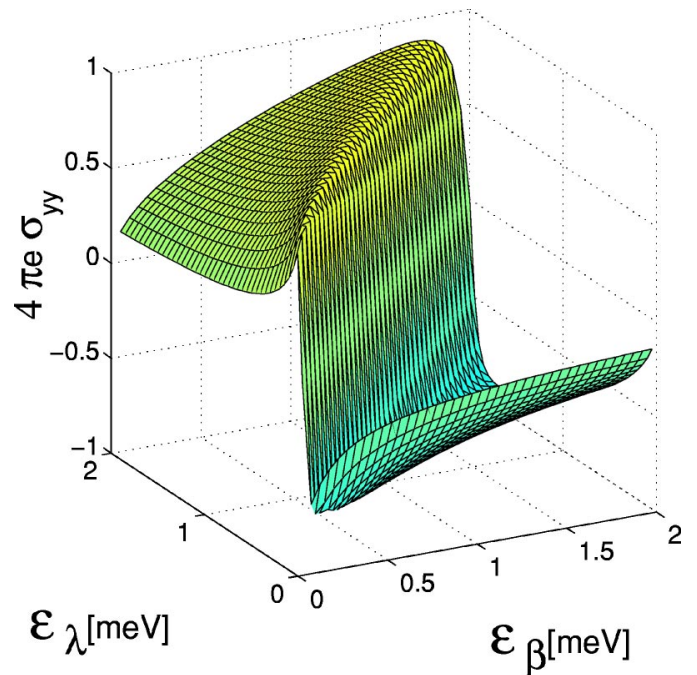

FIG. 1. (Color online) Plot of $\sigma_{y y}^{\text {spin }}$ vs $\epsilon_{\beta}=m^{*} \beta^{2} / \hbar^{2}$ and $\epsilon_{\lambda}$ $=m^{*} \beta^{2} / \hbar^{2}$ with Fermi energy $\epsilon_{F}=50 \mathrm{meV}, \eta=0.63 \mathrm{meV}$, and $m^{*} / m_{e}=0.05$.

conductivity $\sigma_{x y}^{\text {spin }}$ and the spin diagonal conductivity $\sigma_{y y}^{\text {spin }}$ are slightly suppressed for $\epsilon_{F},\left|\epsilon_{\lambda}-\epsilon_{\beta}\right|>\eta$,

$$
\begin{gathered}
\sigma_{x y}^{\text {spin }}=\frac{e}{8 \pi}\left(1-\frac{\eta^{2}\left(\epsilon_{\lambda}+\epsilon_{\beta}\right)}{8 \epsilon_{F}\left(\epsilon_{\lambda}-\epsilon_{\beta}\right)^{2}}\right), \\
\sigma_{y y}^{\text {spin }}=\frac{e}{8 \pi} \sqrt{\frac{\epsilon_{\beta}}{\epsilon_{\lambda}}}\left(1-\frac{\eta^{2} \epsilon_{\lambda}}{4 \epsilon_{F}\left(\epsilon_{\lambda}-\epsilon_{\beta}\right)^{2}}\right),
\end{gathered}
$$

for $\epsilon_{\lambda}>\epsilon_{\beta}$, and for $\epsilon_{\lambda}<\epsilon_{\beta}$ the equations have opposite sign and we must switch $\epsilon_{\beta}$ and $\epsilon_{\lambda}$ in Eq. (13). We note that Eq. (12) is in agreement with Ref. 30 for $\beta=0$. In the strong disorder limit of Eqs. (10) and (11) the spin conductivity tensor goes as $\epsilon_{F} \epsilon_{\lambda / \beta} / \eta^{2}$ but in this case one must still assume $\epsilon_{F} / \eta \gg 1$ (a basic justification of the above approximation) and therefore $\epsilon_{\lambda / \beta} / \eta \ll 1$ is the condition for such effects to vanish. We also note that the specific coefficients in the above expression proportional to $\eta^{2}$ will change since the full expression (9) which takes into account the broadening present in the occupation number must be used in a quantitative analysis. This analytical result has been recently verified numerically in several finite size calculations in the presence of disorder and system sizes a few times larger than the mean free path. ${ }^{33,34}$

In current 2DEG high quality samples $12,13,19,35$ the typical carrier concentrations range from $5 \times 10^{11}$ to $10^{12} \mathrm{~cm}^{-2}$, the $\lambda, \beta$ range is $1-5 \times 10^{-11} \mathrm{eV} \mathrm{m}$, and the mobilities range is $1-50 \mathrm{~m}^{2} / \mathrm{V} \mathrm{s}$. In terms of the effective mass ratio $\mathrm{m}^{*} / \mathrm{m}_{e}$ the energy scales defined above are given by

$$
\begin{gathered}
\epsilon_{F} \approx 0.24 \frac{n_{2 D}\left[10^{11} \mathrm{~cm}^{-2}\right]}{m^{*} / m_{e}}, \\
\epsilon_{\lambda / \beta} \approx 1.31 \frac{m^{*}}{m_{e}}\left(\lambda / \beta\left[10^{-11} \mathrm{eV} \mathrm{m}\right]\right)^{2},
\end{gathered}
$$

$$
\eta \approx \frac{0.116}{\mu[\mathrm{m} / \mathrm{V} \mathrm{s}]\left(m^{*} / m_{e}\right)}
$$

with $\mu$ being in this case the mobility of the sample. The typical $\mathrm{m}^{*} / m_{e}=0.05$ in InAs based heterostructures gives a range of $\epsilon_{F}=20-50 \mathrm{meV}, \epsilon_{\lambda / \beta}=0.07-1.6 \mathrm{meV}$, and $\eta$ $=0.05-2.3 \mathrm{meV}$. Also, the largest mean free path is of the order of $7.8 \mu \mathrm{m}$ and the spin-coherence length is necessarily several times larger. This indicates that current samples (although not all) are already within the regime where these effects should be observable and the weak scattering mesoscopic regime applicable.

It is also important to note that the intrinsic character of our spin Hall effect, compared to the extrinsic character of the effect discussed by Hirsch, ${ }^{9}$ is analogous to the intrinsic contribution to the anomalous Hall effect recently emphasized in various studies, ${ }^{27,29,36-38}$ proposed to be the main contribution to the anomalous Hall effect in some ferromagnets and strongly polarized paramagnets. In both cases scattering contributions to the Hall conductivities can become important if skew scattering ${ }^{39}$ is present, especially when the overall electron scattering rate is small and the steady state distribution function of the current-carrying state is strongly disturbed compared to the equilibrium one. In the case of (III,Mn)V ferromagnetic semiconductors for example, the intrinsic theory of the anomalous Hall effect accounts rather convincingly for experimental observations. ${ }^{36}$

Besides the several approaches already proposed to measure the spin-Hall effect, $, 7,12,37$ the tunability of both the Rashba and Dresselhaus coupling parameters ${ }^{12,13}$ and the non-zero diagonal spin-current generated in the presence of a linear Dresselhaus term offers new possibilities. We propose a bottom gate sample coupled with non-contact probes such as spatially resolved Kerr effect measuremnts, ferromagnetic STM, or scanning Hall probe microscope. Having four noncontact local probe observations (two for Hall and two for diagonal conductivity) on the edges of the sample as a function of gate voltage which varies the Rashba and Dresselhaus coupling ${ }^{12,13}$ simultaneous acquisition of transverse and diagonal spin currents without the usual spurious geometrical effects present in typical transport measurements. Of course, a major challenge is the small resolution needed in order to satisfy the mesoscopic requirement which will only be obtained in the largest mobility samples.

Note added in proof. After the submission of this article the author became aware of a work which independently reached similar conclusions as us within a Berry's phase approach. $^{40}$

The authors would like to thank T. Jungwirth, A. H. MacDonald, S. Murakami, N. Nagaosa, Q. Niu, and S.-C. Zhang for extensive and fruitful discussions, and J. Schliemann and D. Loss for useful correspondence. N.A.S. was supported by DOE under grant No. DE-FG03-96ER45598, NSF under grant DMR0072115 and Telecommunication and Information Task Force at TAMU. 
${ }^{1}$ S.A. Wolf, Science 294, 1488 (2001).

${ }^{2}$ See, for example, F. Meier and D. Loss, Phys. Rev. Lett. 90, 167204 (2003); F. Schuetz, M. Kollar, and P. Kopietz, cond-mat/ 0308230; A.M. Bratkovsky and V.V. Osipov, cond-mat/ 0307656; K.C. Hall et al., Appl. Phys. Lett. 83, 2937 (2003); A. Slobodskyy, C. Grould, T. Slobodskyy, C. Becker, G. Schmidt, and W. Molenkamp, Phys. Rev. Lett. 90, 246601 (2003); C.-R. $\mathrm{Hu}$, cond-mat/0308027.

${ }^{3}$ M.N. Baibich et al., Phys. Rev. Lett. 61, 2472 (1988); G. Binasch et al., Phys. Rev. B 39, 4828 (1989); J.S. Moodera et al., Phys. Rev. Lett. 74, 3273, (1995); W.J. Gallagher et al., J. Appl. Phys. 81, 3741 (1997).

${ }^{4}$ R. Fiederling et al., Nature (London) 402, 787 (1999); G. Schmidt and L.W. Molenkamp, Semicond. Sci. Technol. 17, 310 (2002), and work cited therein; B.T. Jonker, Proc. IEEE 91, 727 (2003).

${ }^{5}$ J.M. Kikkawa and D.D. Awschalom, Phys. Rev. Lett. 80, 4313 (1998).

${ }^{6}$ H. Ohno, Science 281, 951 (1998).

${ }^{7}$ S. Murakami, N. Nagaosa, and S.-C. Zhang, Science 301, 1348 (2003).

${ }^{8}$ J. Sinova, D. Culcer, Q. Niu, N.A. Sinitsyn, T. Jungwirth, and A.H. MacDonald, Phys. Rev. Lett. 92, 126603 (2004).

${ }^{9}$ J.E. Hirsch, Phys. Rev. Lett. 83, 1834 (1999).

${ }^{10}$ S. Zhang, Phys. Rev. Lett. 85, 393 (2000).

${ }^{11}$ M.I. Dyakonov and V.I. Perel, Zh. Eksp. Teor. Fiz. 13, 657 (1971) [Sov. Phys. JETP 33, 467 (1971)].

${ }^{12}$ J.B. Miller, et al., Phys. Rev. Lett. 90, 076807 (2003).

${ }^{13}$ S.D. Ganichev et al., Phys. Rev. Lett. 92, 256601 (2004).

${ }^{14}$ J. Schliemann, J.C. Egues, and D. Loss, Phys. Rev. Lett. 90, 146801 (2003).

${ }^{15}$ Unlike the equilibrium in-plane polarized spin currents mentioned in a recent work by E. Rashba, cond-mat/0311110, the currents which we consider here are polarized perpendicular to the plane and vanish in the zero electric field limit. Hence they arise as a linear response to the perturbation and no other equilibrium current contributions need to be substracted.

${ }^{16}$ E.G. Mishchenko and B.I. Halperin, Phys. Rev. B 68, 045317 (2003).
${ }^{17}$ Y.A. Bychkov and E.I. Rashba, J. Phys. C 17, 6039 (1984).

${ }^{18}$ G. Dresselhaus, Phys. Rev. 100, 580 (1955).

${ }^{19}$ J. Nitta, T. Akazaki, Hideaki Takayanagi, and T. Enoki, Phys. Rev. Lett. 78, 1335 (2003).

${ }^{20}$ S.D. Ganichev et al., Nature (London) 417, 153 (2002).

${ }^{21}$ T. Koga, J. Nitta, T. Akazaki, and H. Takayanagi, Phys. Rev. Lett. 89, 046801 (2002).

${ }^{22}$ S.A. Studenikin, P.R. Coleridge, N. Ahmed, P.J. Poole, and A. Sachrajda, Phys. Rev. B 68, 035317 (2003).

${ }^{23}$ J. Inoue, G.E.W. Bauer, and L.W. Molenkamp, Phys. Rev. B 67, 033104 (2003).

${ }^{24}$ J. Schliemann and D. Loss, Phys. Rev. B 68, 165311 (2003).

${ }^{25}$ S. Datta and B. Das, Appl. Phys. Lett. 56, 665 (1990).

${ }^{26}$ D. Culcer et al., Phys. Rev. Lett. 93, 046602 (2004).

${ }^{27}$ G. Sundaram and Q. Niu, Phys. Rev. B 59, 14915 (1999).

${ }^{28}$ N.W. Ashcroft and N.D. Mermin, Solid State Physics, 2nd ed. (Saunders College Publishing, Philadelphia, 1976).

${ }^{29}$ J. Sinova, T. Jungwirth, J. Kucera, and A.H. MacDonald, Phys. Rev. B 67, 235203 (2003).

${ }^{30}$ J. Schliemann and D. Loss, Phys. Rev. B 69, 165315 (2004).

${ }^{31}$ J. Inoue, G.E.W. Bauer, and L.W. Molenkamp, Phys. Rev. B 70, 041303 (2004).

${ }^{32}$ G. Mahan, Many-Particle Physics, 2nd ed. (Plenum, New York, 1993).

${ }^{33}$ Y. Xiong and X.C. Xie, cond-mat/0403083.

${ }^{34}$ K. Nemura, J. Sinova, Q. Niu, and A.H. MacDonald (unpublished).

${ }^{35}$ D. Grundler, Phys. Rev. Lett. 84, 6074 (2000).

${ }^{36}$ T. Jungwirth, Q. Niu, and A.H. MacDonald, Phys. Rev. Lett. 88, 207208 (2002); T. Jungwirth et al., Appl. Phys. Lett. 83, 320 (2003).

${ }^{37}$ D. Culcer, A.H. MacDonald, and Q. Niu, Phys. Rev. B 68, 045327 (2003).

${ }^{38}$ K. Ohgushi, S. Murakami, and N. Nagaosa, Phys. Rev. B 62, R6065 (2000); M. Onoda and N. Nagaosa, J. Phys. Soc. Jpn. 71, 19 (2002).

${ }^{39}$ A. Crépieux et al., cond-mat/0105614; T. Dietl et al., cond-mat/ 0306484.

${ }^{40}$ S.-Q. Shen, Phys. Rev. B 70, 081311 (2004). 